\title{
Spin-torque generation by dc or ac voltages in magnetic layered structures
}

\author{
F. Romeo ${ }^{1}$, and R. Citro ${ }^{1,2}$ \\ ${ }^{1}$ Dipartimento di Fisica "E. R. Caianiello" and C.N.I.S.M., \\ Università degli Studi di Salerno, Via S. Allende, I-84081 Baronissi (Sa), Italy \\ ${ }^{2}$ Laboratorio Regionale INFM-CNR SuperMat, Via S. Allende, I-84081 Baronissi (Sa), Italy
}

\begin{abstract}
A general expression of the current induced spin torque in a magnetic layered structure in the presence of external dc or ac voltages is derived in the framework of the scattering matrix approach. A detailed analysis is performed for a magnetic-nonmagnetic-magnetic trilayer connected to external leads in the presence of dc voltage bias in the ballistic regime. Alternatively, the possibility of producing spin torque by means of the adiabatic ac modulation of external gate voltages (quantum pumping) is proposed and discussed.
\end{abstract}

PACS numbers: 73.23.-b,72.25.Pn,75.60.Jk,72.15.Qm

\section{INTRODUCTION}

Multilayers of alternating magnetic (generally ferromagnetic) and nonmagnetic metal layers have recently attracted a lot of attention because of giant magnetoresistance (GMR) effects. In fact their electrical resistance depends strongly on whether the moments of adjacent magnetic layers are parallel or antiparallel and this effect has allowed the development of new kinds of magnetic memory devices $\underline{\underline{1}}$ The origin of the GMR effect is the stronger scattering of conduction electrons by a magnetic layer when their spins lie antiparallel to the layers magnetic moment compared to the case when their spins are parallel to the moment. Thus the orientations of magnetic moments can affect the flow of electrons, but as a reciprocal effect a polarized electron current scattering from a magnetic layer can affect the moment of the layer itself. In fact, as proposed by Berger ${ }^{2}$ and Slonczewski, $\underline{3}$ an electric current passing perpendicularly through a magnetic multilayer may exert a torque on the moments of the magnetic layers. This effect is known as "spin transfer, and may alter the magnetization state of the layer. Of course this is a different mechanism from the effects of current induced magnetic fields and offers the possibility of realizing new kinds of magnetic devices. Importantly, it can serve as a mean to detect the spin dynamics at nanometer scale and measure spin currents. However, in order to utilize these effects in real devices, it is necessary to achieve a quantitative understanding of spin-current-induced torques and also provide different ways for their generation.

In the present literature a semiclassical WKB approximation with spin dependent potentials in 1D has been proposed in Ref. 3]. A generalization of this study has appeared in Ref.[4] and an extension of this calculation to take into account the band structure effects on the degree to which an electron is transmitted through a magnetic/nonmagnetic interface has been proposed by Brataas et al. $\frac{5}{}$ by means of the kinetic equations for spin currents and by Waintal et al $\underline{6}$ by using a LandauerBüttiker type of approach. A first principle theory based on the spin mixing conductance appeared in [7].
Here we present a general formalism based on the scattering matrix approach to calculate the torques in a magnetic layered structure in the ballistic regime. Then we use this formalism to make an explicit calculation for a magnetic(M)-nonmagnetic(NM)-magnetic(M) trilayer connected to two metallic external leads. In this case the ballistic regime is characterized by a spin-diffusion length $l_{s}$ and a mean-free-path $l_{m}$ larger than the NM spacer. We perform first the calculation in the presence of a dc voltage bias applied to the leads, or equivalently for currents perpendicular to the microstructure, then we proposed an alternative way of achieving a spin-torque by the adiabatic ac modulation of two system parameters (in our case the barrier heights at the M-NM interface), i.e. by a quantum pump mechanism ${ }^{-}$. Although we limit our calculation to the single channel case, differently from Ref $[6$ all spin-scattering processes at the M-NM interface are properly taken into account, including spin-flip mechanisms.

The organization of the paper is the following: In Sec III we introduce the model Hamiltonian and the general expression for the spin-torque. In Sec III we develop the scattering matrix approach generalized for the calculation of the spin-torque in a magnetic layered structure. In Sec IV we discuss the adiabatic quantum pumping of spin-torque and finally in Sec $\nabla$ we present the results for a specific system, a trylayer of M-NM-M metals connected to two nonmagnetic leads. A quantitative analysis of the spin-torque components is presented both in the case of a dc bias applied to the external leads and when a quantum pump mechanism is activated. The two ways of generating a spin-torque presents some differences that are comparatively discussed. These differences could be important for the realization of new magnetic devices.

\section{THE MODEL AND FORMALISM}

Let us consider a $1 \mathrm{D}$ system whose Hamiltonian is: $H=-\frac{\hbar^{2}}{2 m} \partial_{x}^{2}+V(x, \vec{\sigma})$, where $V(x, \vec{\sigma})$ is some spindependent potential operator. The field operator describing the electron state $\Psi$, obeys the Schrödinger equation 
$\vec{H} \Psi=i \hbar \partial_{t} \Psi$ and $\Psi^{\dagger} \overleftarrow{H}=-i \hbar \partial_{t} \Psi^{\dagger}$ and the time evolution of the electron charge density $\rho=\Psi^{\dagger} e \Psi$ is determined by the Heisenberg equation:

$$
\partial_{t}\left(\Psi^{\dagger} e \Psi\right)=\frac{i e}{\hbar}\left[\Psi^{\dagger}(\overleftarrow{H}-\vec{H}) \Psi\right]=-\partial_{x} J
$$

where $J=\Psi^{\dagger} e \hat{v} \Psi$ is the current density, while $\hat{v}=$ $\frac{i \hbar}{2 m}\left(\overleftarrow{\partial}_{x}-\vec{\partial}_{x}\right)$ is the velocity operator along the $x$ direction. From (1) the well known continuity equation $\partial_{t} \rho+\partial_{x} J=0$ is obtained.

In an analogous manner, the time evolution of the spin density $S_{\mu}=\Psi^{\dagger} \frac{\hbar}{2} \sigma_{\mu} \Psi, \mu=\{x, y, z\} \quad\left(\sigma_{\mu}\right.$ are the Pauli matrices), can be derived:

$$
\partial_{t} S_{\mu}=-\partial_{x} J_{\mu}^{s}+\tau_{\mu} .
$$

Here $J_{\mu}^{s}=\Psi^{\dagger} \frac{\hbar}{2} \sigma_{\mu} \hat{v} \Psi$ is the $\mu$-th component of the spin current density and $\tau_{\mu}$ is the the density of spin torque. When the spin-dependent potential describes the Zeeman interaction between the spin and a magnetic field along the direction $\hat{n}(x), V(x, \vec{\sigma})=\gamma(x) \hat{n}(x) \cdot \vec{\sigma}$, the density of spin torque is explicitly given by $\tau_{\mu}=\gamma(x) \Psi^{\dagger}(\hat{n}(x) \times$ $\vec{\sigma})_{\mu} \Psi=\frac{2 \gamma(x)}{\hbar}(\hat{n}(x) \times \vec{S})_{\mu}$. When $\gamma(x)=0$, the spin torque term vanishes and a continuity equation is obeyed by the spin currents, $\partial_{t} \vec{S}+\partial_{x} \vec{J}^{s}=0$. In the case of a system in which a magnetic central region $x \in[-a, a]$ is connected to two external non-magnetic leads, under the stationary condition for the spin-current (i.e. $\partial_{t} \vec{S}=0$ ) the spin torque per unit of area can be calculated by spatial integration of the equation $\partial_{x} J_{\mu}^{s}=\tau_{\mu}$ along the $x$-direction:

$$
\vec{J}_{R}^{s}-\vec{J}_{L}^{s}=\int_{-a}^{+a} d x \vec{\tau}=\vec{T},
$$

where $\vec{J}_{R / L}^{s}=\vec{J}^{s}( \pm \infty)$ is the value of the current at infinity. The above result tells that the spin torque per unit of area is due the difference of the spin currents on the right and on the left of the magnetic scattering region. In the following Section we analyze the spin torque transfer within a scattering matrix approach.

\section{DENSITY OF SPIN CURRENT WITHIN THE SCATTERING APPROACH}

In order to calculate the spin torque through a magnetic region we employ the scattering matrix formalism of Ref.[10] and consider a single channel approximation to simplify the derivation.

The $S$-matrix connects the outgoing states to the incoming states through the relation:

$$
b_{\sigma}^{\alpha}=\sum_{\sigma^{\prime} \beta} S_{\sigma \sigma^{\prime}}^{\alpha \beta} a_{\sigma^{\prime}}^{\beta},
$$

where $b_{\sigma}^{\alpha}, a_{\sigma}^{\alpha}$ are the scattering operators for the outgoing and incoming states, $\sigma= \pm$ is the spin index, $\alpha=\{$ left,right $\}$ represents the lead index, while the $S$-matrix must be unitary, i.e. $S^{\dagger} S=1$. The quantum field representing the electron state in the lead $\alpha$ can be expressed in terms of the scattering operators as follows:

$$
\begin{aligned}
& \Psi_{\alpha}(x, t)=\sum_{\sigma} \int d E \rho_{\alpha}(E) \exp \left[-i \frac{E}{\hbar} t\right]|\sigma\rangle \times \\
& {\left[e^{i k x} a_{\sigma}^{\alpha}(E)+e^{-i k x} b_{\sigma}^{\alpha}(E)\right],}
\end{aligned}
$$

where $\rho_{\alpha}(E)=\left[\sqrt{2 \pi \hbar v_{\alpha}(E)}\right]^{-1}$ is the density of states of lead $\alpha, v_{\alpha}(E)$ is the velocity of the electrons with wave vector $k=\sqrt{2 m E} / \hbar$ and the velocity is chosen to have positive orientation for the incoming states. The spincurrent density $\vec{J}^{s}=\Psi^{\dagger} \frac{\hbar}{2} \vec{\sigma} \hat{v} \Psi$ can be calculated with the approach of Ref $\frac{10}{1}$ and the result is:

$$
\begin{aligned}
& J_{\mu, \alpha}^{s}=\frac{1}{4 \pi} \int d E d E^{\prime} \exp \left[i \frac{\left(E-E^{\prime}\right)}{\hbar} t\right] \times \\
& {\left[a^{\alpha \dagger}(E) \sigma_{\mu} a^{\alpha}\left(E^{\prime}\right)-b^{\alpha \dagger}(E) \sigma_{\mu} b^{\alpha}\left(E^{\prime}\right)\right],}
\end{aligned}
$$

where the following spinorial representation has been introduced:

$$
a^{\alpha}=\left(\begin{array}{c}
a_{+}^{\alpha} \\
a_{-}^{\alpha}
\end{array}\right)
$$

while $a^{\alpha \dagger}=\left(a_{+}^{\alpha \dagger}, a_{-}^{\alpha \dagger}\right)$. Using the relation $\left\langle a_{\sigma}^{\alpha \dagger}(E) a_{\sigma^{\prime}}^{\beta}\left(E^{\prime}\right)\right\rangle=\delta_{\alpha \beta} \delta_{\sigma \sigma^{\prime}} f_{\beta}(E), f_{\beta}(E)$ being the Fermi function of the lead $\beta$ and Eq.(44), the $\mu$-th component of the spin density current $\left\langle J_{\mu, \alpha}^{s}\right\rangle$ in the lead $\alpha$ can be written as $\stackrel{9}{=}$ :

$\left\langle J_{\mu, \alpha}^{s}\right\rangle=-\frac{1}{4 \pi} \sum_{\beta} \int d E \operatorname{Tr}\left\{S^{\alpha \beta \dagger}(E) \sigma_{\mu} S^{\alpha \beta}(E)\right\} f_{\beta}(E),(8)$

while using ( 3) the spin torque per unit of area $T_{\mu}=$ $\int d x \tau_{\mu}$ is:

$$
T_{\mu}=\frac{1}{4 \pi} \sum_{\alpha \beta=L, R} \int d E T r\left\{S^{\alpha \beta \dagger}(E) \sigma_{\mu} S^{\alpha \beta}(E)\right\} f_{\beta}(E) \text {. (9) }
$$

When a dc voltage bias $V$ is applied to the external leads, changing their chemical potentials in $\mu_{l, r}=E_{F} \pm \mathrm{eV} / 2$, the variation of the spin-torque with respect to the external perturbation $w=e V / 2$ can be written as $\delta_{w} T_{\mu}=$ $\partial_{w} T_{\mu} \delta w$ in the linear response regime. It is determined by the torkance $\partial_{w} T_{\mu}$. Using Eq.(9) and taking the zero temperature limit, we explicitly have $\mathrm{e}^{\underline{11}}$ :

$$
\partial_{w} T_{\mu}=\left.\frac{1}{2 \pi} \operatorname{Tr}\left\{\sigma_{\mu}\left(S^{21} S^{21 \dagger}-S^{12} S^{12 \dagger}\right)\right\}\right|_{E=E_{F}} .
$$

The torkance along the direction of the unit vector $\hat{n}=$ $\left(n_{x}, n_{y}, n_{z}\right)$ is obtained as $\hat{n} \cdot \partial_{w} \vec{T}$. Eq.(10) describes the magnetic answer of the system to an external dc voltage. Let us note that compared to previous works 3.6 our calculation of the scattering matrix includes also off-diagonal spin-flip reflection and transmission amplitudes. 


\section{ALL ELECTRICAL GENERATION OF SPIN TORQUE BY QUANTUM PUMPING}

In this section we discuss the generation of spin torque by means of ac external gate voltages. In particular we generalize the notion of quantum pumping of charges due to Thouless ${ }^{8}$ to the spin torque. In a quantum pump a dc particle current is generated by the ac adiabatic modulation of at least two out-of-phase independent parameters of the system (e.g. local magnetic fields or gate voltages) in absence of bias. In our calculation we will show that since pumping procedure in a magnetic layered structure can generate spin currents other than charge currents, a spin-torque is generated by the gradient of spin current (see Eqs.(2)-(3)). In particular, we focus on the system of Fig.(11) in which a microstructure made of a central non-magnetic-region (NM) is connected to two external non-magnetic leads (LL and RL) through magnetic layers (M1 and M2) (whose width is taken less than the De Broglie wavelength). Applying the idea of pumping, we modulate in time the barriers heights at the interface between the M-NM regions by the top gates G1 and G2. In the presence of this ac modulation the scattering matrix $S$ depends explicitly on time and the relation between incoming and outgoing states is:

$$
b^{\alpha}(t)=\sum_{\beta} \int d t^{\prime} S^{\alpha \beta}\left(t, t^{\prime}\right) a^{\beta}\left(t^{\prime}\right),
$$

where the spin-indices are absorbed in the spinorial notation (see Eq.(7)). When the gates are varied adiabatically in time, an instantaneous approximation can be made, i.e. $S^{\alpha \beta}\left(t, t^{\prime}\right)=\delta\left(t-t^{\prime}\right) S^{\alpha \beta}(t)$, and thus $b^{\alpha}(t)=\sum_{\beta} S^{\alpha \beta}(t) a^{\beta}(t)$. In particular, since the time dependence of the scattering matrix $S(t)$ is induced by two external parameters of the form

$$
\begin{aligned}
& X_{1}(t)=X_{1}^{0}+X_{1}^{\omega} \sin (\omega t) \\
& X_{2}(t)=X_{2}^{0}+X_{2}^{\omega} \sin (\omega t+\varphi),
\end{aligned}
$$

then $S(t)=S\left(X_{1}(t), X_{2}(t)\right)$. In particular, when the amplitude of the ac parameters is small, i.e. $X_{1,2}^{\omega} \ll X_{1,2}^{0}$, the scattering matrix can be expanded as follows:

$$
S^{\alpha \beta}(t) \simeq S_{0}^{\alpha \beta}+\sum_{\eta= \pm 1} s_{\eta}^{\alpha \beta} e^{i \eta \omega t},
$$

where $\omega=2 \pi \nu$ is the angular frequency of the adiabatic modulation and the matrices $s_{\eta}^{\alpha \beta}$ are given by

$$
s_{\eta}=-\frac{i \eta}{2}\left[X_{1}^{\omega}\left(\partial_{X_{1}} S\right)_{0}+X_{2}^{\omega} e^{i \eta \varphi}\left(\partial_{X_{2}} S\right)_{0}\right] .
$$

The Fourier transform of (13) is then:

$$
S^{\alpha \beta}(E)=2 \pi\left[S_{0}^{\alpha \beta} \delta(E)+\sum_{\eta= \pm 1} s_{\eta}^{\alpha \beta} \delta(E+\eta \omega)\right] .
$$

Consequently, the relation between the outgoing and incoming states in the Fourier space takes the following

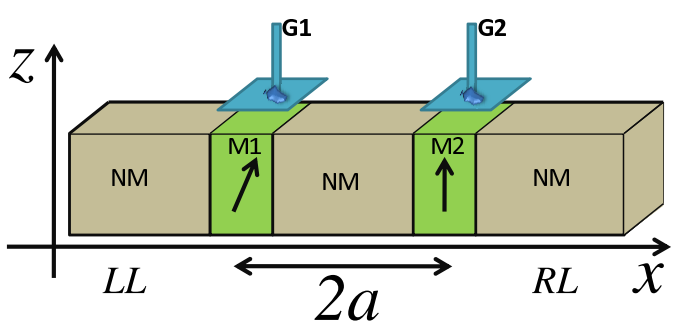

FIG. 1: Schematic representation of the NM/M/NM/M/NM system described in the main text.

form:

$$
b^{\alpha}(E)=\sum_{\beta}\left[S_{0}^{\alpha \beta} a^{\beta}(E)+\sum_{\eta} s_{\eta}^{\alpha \beta} a^{\beta}(E+\eta \omega)\right] .
$$

Using Eq.(16) in (6) the $\mu$-component of the spin torque per unit of area is given by:

$$
T_{\mu}=\frac{1}{4 \pi} \sum_{\eta \alpha \beta} \int d E T r\left\{s_{\eta}^{\alpha \beta \dagger} \sigma_{\mu} s_{\eta}^{\alpha \beta}\right\} f_{\beta}(E+\eta \omega) .
$$

Since no bias is present between the leads $f_{\beta}(E)=f(E)$, and in the zero temperature limit, the $\mu$-th component of spin-torque per unit area to leading order in the adiabatic frequency $\omega$ is:

$$
T_{\mu}=-\frac{\hbar \omega}{4 \pi} \sum_{\alpha \beta \eta} \eta \operatorname{Tr}\left\{\sigma_{\mu} s_{\eta}^{\alpha \beta} s_{\eta}^{\alpha \beta \dagger}\right\}
$$

(all terms independent from the external perturbation have been dropped). Using (14) this can be rewritten in terms of the parametric derivatives of the scattering matrix as :

$$
T_{\mu}=\frac{\hbar \omega X_{1}^{\omega} X_{2}^{\omega} \sin (\varphi)}{8 \pi} \sum_{\alpha \beta} \operatorname{Tr}\left\{A_{\mu}^{\alpha \beta}+A_{\mu}^{\alpha \beta \dagger}\right\},
$$

where we introduced the quantity $A_{\mu}^{\alpha \beta}=$ $i\left(\partial_{X_{2}} S^{\alpha \beta \dagger}\right)_{0} \sigma_{\mu}\left(\partial_{X_{1}} S^{\alpha \beta}\right)_{0}$. The torque generated in the direction of the unit vector $\hat{n}$ is given by $T_{\|}=\hat{n} \cdot \vec{T}$. Eq.(19) represents the torque pumped in a magnetic system and in principle could be very different from the one induced by a dc voltage. In the following we perform the explicit calculation for the system of Fig. 1.

\section{SPIN TORQUE IN M-NM-M SYSTEMS}

Here we analyze the spin torque generated in the system of Fig. 1 in the following two situations: (i) in the presence of a dc voltage applied to the external leads; (ii) in the absence of bias and using a quantum pumping procedure. The central NM region is connected to two external leads via the thin magnetic layers M1 and M2 and without loss of generality we choose the magnetization of M2 parallel to the $z$-axis, while the magnetization 
of the region M1 has arbitrary direction. The distance between the two magnetic barriers is taken $2 a$ and their height is controlled by the the top gates G1 and G2. A minimal model for the system above is given by the following Hamiltonian:

$$
H=-\frac{\hbar^{2}}{2 m} \partial_{x}^{2}+V_{m}(x, \vec{\sigma})+U(x),
$$

where $U(x)=g_{1} \delta(x+a)+g_{2} \delta(x-a)$ is the barriers potential at the interface between M-NM region, while $V_{m}(x, \vec{\sigma})=\gamma_{l} \delta(x+a) \hat{n} \cdot \vec{\sigma}+\gamma_{r} \delta(x-a) \sigma_{z}$ represents the magnetic interaction between the conduction electrons spin and the magnetic moment of the layers M1 and M2. The scattering problem for the Hamiltonian above can be easily solved by imposing the appropriate boundary conditions on the wavefunctions and their derivatives at the interfaces (see, for instance, Ref. 12]) and from the knowledge of the scattering matrix the spin torque $T_{\mu}$ can be calculated using the equations of the previous sections. The following consideration is in order here: The presence of two magnetic layers has the effect of allowing for multiple scattering of the electrons between them, which gives rise to an explicit asymmetry of the spin current and produces a finite spin-torque effect.

In the following we employ the adimensional quantities: $z_{1}=\frac{2 m g 1}{\hbar^{2} k_{F}}, z_{2}=\frac{2 m g 2}{\hbar^{2} k_{F}}, \Gamma_{R}=\frac{2 m \gamma_{r}}{\hbar^{2} k_{F}}, \Gamma_{L}=\frac{2 m \gamma_{l}}{\hbar^{2} k_{F}}$, where $k_{F}$ is the Fermi wavelength, $m=\alpha m_{e}$ is the effective electron mass, while $\alpha$ represents the ratio between the effective and the bare electron mass $m_{e}$. The distances are made adimensional by multiplying by $k_{F}$, i.e. $a \rightarrow k_{F} a$.

\section{A. Spin torque by means of dc voltage bias}

Here we present the results of the spin torque per unit of area generated by a small dc voltage bias $V$ applied to the external leads. In the following we use polar coordinates for the magnetic moment $\vec{M}_{1}=M_{1} \hat{n}$, $\hat{n}=(\sin (\theta) \cos (\phi), \sin (\theta) \sin (\phi), \cos (\theta))$, and assume that the momenta $\vec{M}_{1}$ and $\vec{M}_{2}$ lie in the plane $x-z$. In this case we have $\hat{n}=(\sin (\theta), 0, \cos (\theta))$, where the $\theta$ represents the angle between $\vec{M}_{1}$ and $\vec{M}_{2}$.

In Fig.(2) the three components of the torque (in unit of $e V / 2$ ) are shown as a function of angle $\theta$ between the magnetic momenta of the regions M1 and M2 and by fixing the remaining parameters as follows: $a=2, z_{1}=0.5$, $z_{2}=1, \Gamma_{L}=0.1, \Gamma_{R}=1$.

Similarly to other works ${ }^{6}$, we find that all the torque components vanish when M1 and M2 are parallel or antiparallel. Furthermore, the $z$-component $T_{z}$ of the torque is very small compared to $T_{x}$ and $T_{y}$, while the component perpendicular to the plane of the magnetic moment $T_{y}$ is the strongest. This component of the torque follows a $\sin \theta$ behavior for the specific values of magnetic interactions considered in the figure $\left(\Gamma_{R} \gg \Gamma_{L}\right)$. In fact when the non-spin-polarized electrons are incident on the

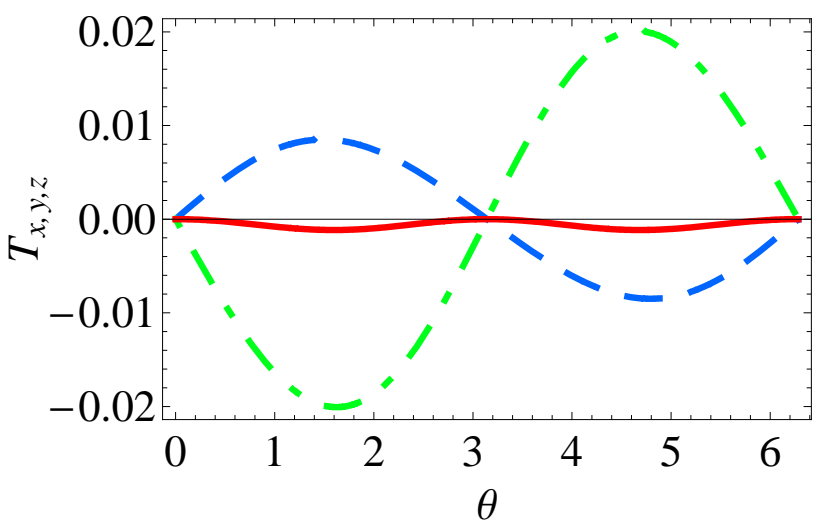

FIG. 2: Torque $T_{x}$ (dashed line), $T_{y}$ (dashed-dotted line), $T_{z}$ (full line) in unit of $e V / 2$ computed as a function of the angle $\theta$ between M1 and M2. The parameters have been fixed as follows: $a=2, z_{1}=0.5, z_{2}=1, \Gamma_{L}=0.1, \Gamma_{R}=1$. When the magnetization directions of the layers M1 and M2 are parallel or anti-parallel the spin torque vanishes.

magnetic layer M2, spin-filtering removes the component of the spin angular momentum perpendicular to the layer moment from the current acting as a polarizer along the $z$ direction. Thus the polarized electrons scattered off M2 and incident on M1 generate an effective torque on the layer momentum M1 proportional to the $\sin \theta$. The negative sign of the spin torque component $T_{y}$ is due to the fact that electrons with spins parallel to the moment of the magnetic region M2 have a larger transmission probability compared to those antiparallel.

In Fig.(3) the components of the torque are shown as a function of the semi-distance $a$ between the gates G1 and G2 and by fixing the remaining parameters as follows: $\theta=1.5, z_{1}=0.5, z_{2}=1, \Gamma_{L}=0.1, \Gamma_{R}=1$. For a mesoscopic system characterized by a De Broglie wavelength $\lambda \approx 20-30 \mathrm{~nm}$ the adimensional distance $a=1$ would correspond to $3.18-4.78 \mathrm{~nm}$. As shown in the figure, the torque presents a characteristic oscillatory behavior with the semi-length of the central region $a$. These oscillations can be regarded as a quantum-size effect. They reflect the perfect ballistic regime of electron transport through the spin valve. The physical mechanism behind the oscillations is the interference effect of the electrons propagating across the non-magnetic-magnetic interface from the right lead to the left lead and electrons propagating backwards. The particular value of the oscillation follows from the values of the spin-dependent Fermi wavevector.

In Fig.(41) we plot $T_{x, y, z}$ as a function of the strength $z_{2}$ of the right barrier (controllable via the gate G2) by fixing the other parameters as: $\theta=1.5, z_{1}=0.5, a=2$, $\Gamma_{L}=0.1, \Gamma_{R}=1$. The torque of course depends crucially on the transparency of the barrier and thus the components $T_{x, y}$ decreases with increasing barrier height becoming comparable to $T_{z}$. An interesting feature appears by increasing the Zeeman interaction $\Gamma_{L}$ as shown in Fig.(5): for values of $\Gamma_{L}$ above 0.2 the $z$-component 


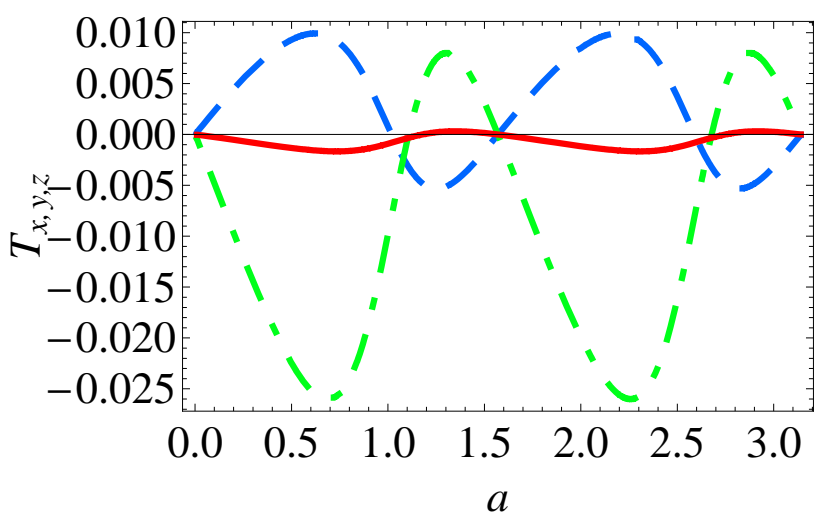

FIG. 3: Torque $T_{x}$ (dashed line), $T_{y}$ (dashed-dotted line), $T_{z}$ (full line) in unit of $e V / 2$ computed as a function of the semidistance $a$ between the gates G1 and G2. The parameters have been fixed as follows: $\theta=1.5, z_{1}=0.5, z_{2}=1, \Gamma_{L}=$ $0.1, \Gamma_{R}=1$. Notice that the torque $T_{y}$, i.e. the torque in the direction perpendicular to the plane $x-z$ presents large oscillations compared with the in-plane components.

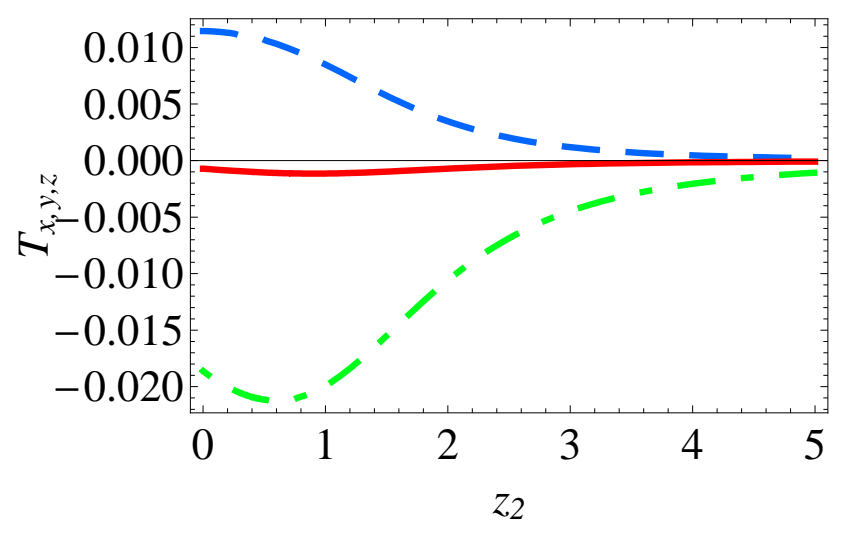

FIG. 4: Torque $T_{x}$ (dashed line), $T_{y}$ (dashed-dotted line), $T_{z}$ (full line) in unit of $e V / 2$ computed as a function of the non-dimensional barrier strength $z_{2}$ controlled by G2. The parameters have been fixed as follows: $\theta=1.5, z_{1}=0.5$, $a=2, \Gamma_{L}=0.1, \Gamma_{R}=1$.

of the torque $T_{z}$ starts to assume relevant values. In fact the magnetic interaction becomes more effective in aligning the electron spins along the magnetic moment of the layer M1 thus reducing the contribution to the gradient of spin current.

\section{B. Spin torque generation by means of adiabatic quantum pumping}

An alternative way to generate a spin torque is the adiabatic quantum pumping technique. As explained above we keep the two external leads at the same chemical potential and in order to generate a spin current we modulate out-of-phase in time the two barrier heights $z_{1}$ and

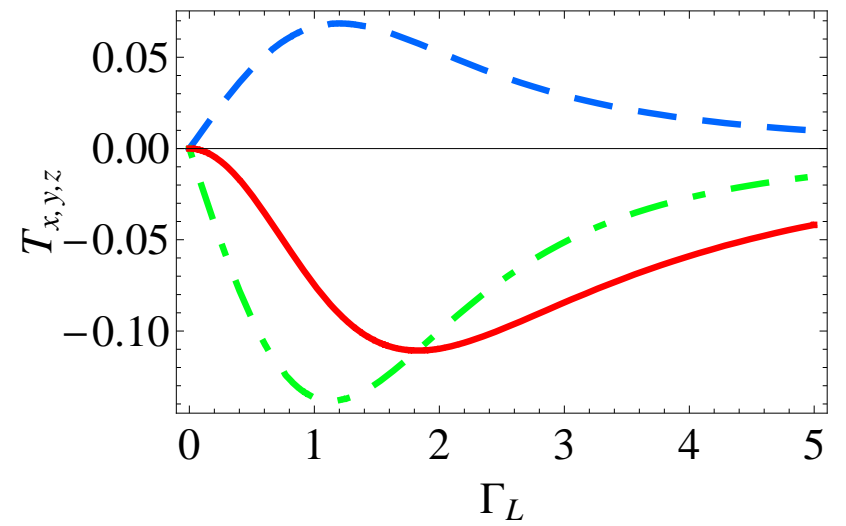

FIG. 5: Torque $T_{x}$ (dashed line), $T_{y}$ (dashed-dotted line), $T_{z}$ (full line) in unit of $e V / 2$ computed as a function of $\Gamma_{L}$ controlled by the magnetic momentum in the region M1. The remaining parameters have been fixed as follows: $\theta=1.5$, $z_{1}=0.5, z_{2}=0.8, a=2, \Gamma_{R}=1$.

$z_{2}$ by the gate voltages $\mathrm{G} 1$ and $\mathrm{G} 2$ :

$$
\begin{aligned}
& z_{1}(t)=z_{1}^{0}+z_{1}^{\omega} \sin (\omega t) \\
& z_{2}(t)=z_{2}^{0}+z_{2}^{\omega} \sin (\omega t+\varphi) .
\end{aligned}
$$

Thus by using (19) the $\mu-t h$ component of the torque in the adiabatic regime is given by:

$$
T_{\mu}=\frac{\hbar \omega z_{1}^{\omega} z_{2}^{\omega} \sin (\varphi)}{8 \pi} \sum_{\alpha \beta} \operatorname{Tr}\left\{A_{\mu}^{\alpha \beta}+A_{\mu}^{\alpha \beta \dagger}\right\}
$$

In the weak pumping regime (i.e. $z_{i}^{0} \gg z_{i}^{\omega}$ ) considered here, $T_{\mu}$ presents a $\sin (\varphi)$ behavior with respect to the pumping phase $\varphi$ and thus in the following analysis we set $\varphi=\pi / 2$ and measure the spin torque in unit of $\hbar \nu / 2$. Concerning the frequencies of the pump, we can safely consider as adiabatic the frequencies for which $\hbar \omega$ is smaller than the first gap in the electron energy spectrum such that the system lies in its ground state ${ }^{8}$. For simplicity, by considering the energy spectrum of a particle confined in a box, we easily find that the threshold frequency is $\tilde{\nu} \sim \frac{h}{2 m^{\star} L^{2}}$, where $L$ is the length of the system, while $m^{\star}=\alpha m_{e}$ is the effective mass of the electrons. Thus for a system with $L=1.5 \mu \mathrm{m}$ and effective mass ratio $\alpha=0.023, \tilde{\nu} \approx 43.4 \mathrm{GHz}$ which is consistent with the values of the frequencies of quantum pumping in quantum dots. For a smaller system characterized by $L=0.5 \mu \mathrm{m}$ and $\alpha=0.023$ we obtain $\tilde{\nu} \approx 396.5 \mathrm{GHz}$ which is a more convenient value to achieve in experiments.

In Fig.(6) we plot the components $T_{\mu}$ as a function of the angle $\theta$ between the magnetization M1 and M2 and by fixing the other parameters as follows: $z_{1}^{\omega}=z_{2}^{\omega}=0.2$, $\Gamma_{L}=0.9, z_{1}^{0}=0.5, z_{2}^{0}=0.3, a=1, \Gamma_{R}=1$. Compared to the case of dc bias, the $\theta$ dependence of the torque is not a simple $\sin \theta$ form because the strength of the magnetic interactions $\Gamma_{L}$ and $\Gamma_{R}$ is comparable and thus both $\cos \theta$ and $\sin \theta$ terms contribute. Moreover as 


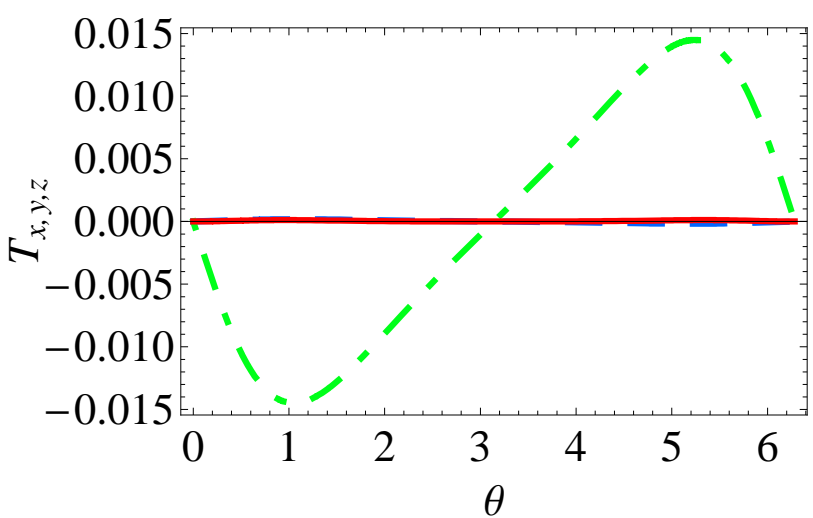

FIG. 6: Torque $T_{x}$ (dashed line), $T_{y}$ (dashed-dotted line), $T_{z}$ (full line) in unit of $\hbar \nu / 2$ computed as a function of $\theta$ between M1 and M2. The remaining parameters have been fixed as follows: $z_{1}^{\omega}=z_{2}^{\omega}=0.2, \Gamma_{L}=0.9, z_{1}^{0}=0.5, z_{2}^{0}=0.3, a=1$, $\Gamma_{R}=1$.

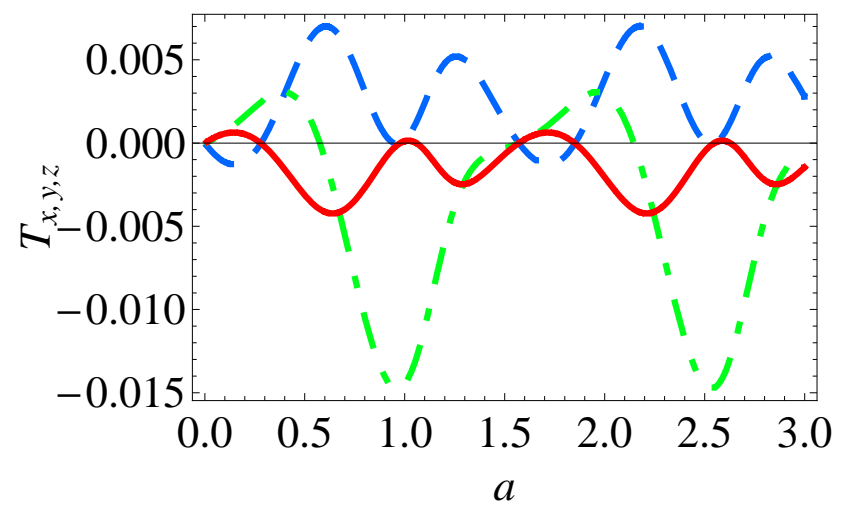

FIG. 7: Torque $T_{x}$ (dashed line), $T_{y}$ (dashed-dotted line), $T_{z}$ (full line) in unit of $\hbar \nu / 2$ computed as a function of the semi-distance $a$ between the gates G1 and G2. The remaining parameters have been fixed as follows: $z_{1}^{\omega}=z_{2}^{\omega}=0.2, \Gamma_{L}=$ $0.9, z_{1}^{0}=0.5, z_{2}^{0}=0.3, \Gamma_{R}=1, \theta=1$.

in the previous analysis, while the in-plane component of the spin torque $T_{x}$ and $T_{z}$ assume very small values in the parameter region considered, the $T_{y}$ component presents relevant values. Indeed, by setting $\theta \approx 1$ and for pumping frequencies $\nu$ of the order of $10-30 \mathrm{GHz}$ we have a torque component $T_{y} \approx 0.05-0.15 \mu \mathrm{eV}$ per unit of area, which is of the same order of magnitude obtained in a similar system under dc bias (see Ref.[13]). In Fig.(7) we show the spin torque components $T_{x, y, z}$ as a function of the semi-distance $a$ between the gates G1 and G2, for the remaining parameters: $z_{1}^{\omega}=z_{2}^{\omega}=0.2$, $\Gamma_{L}=0.9, z_{1}^{0}=0.5, z_{2}^{0}=0.3, \Gamma_{R}=1, \theta=1$. Compared to the case in which the spin-torque is generated by an external dc bias, more harmonics are present in the torque dependence on $a$. This behavior can be qualitatively explained by the fact that the torque generated by the pumping procedure is related to the parametric derivatives of the scattering matrix. In Fig.(8) we show

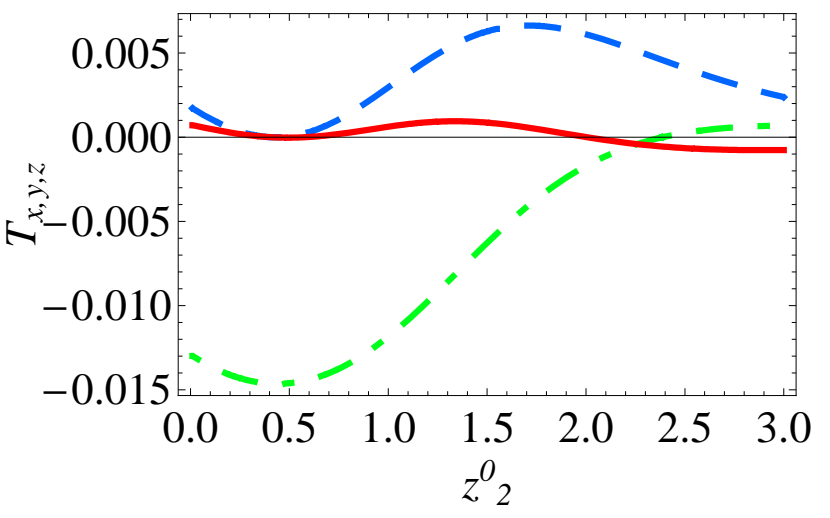

FIG. 8: Torque $T_{x}$ (dashed line), $T_{y}$ (dashed-dotted line), $T_{z}$ (full line) in unit of $\hbar \nu / 2$ computed as a function of the static part of the non-dimensional barrier strength $z_{2}^{0}$ controlled by G2. The remaining parameters have been fixed as follows: $z_{1}^{\omega}=z_{2}^{\omega}=0.2, \Gamma_{L}=0.9, z_{1}^{0}=0.5, a=1, \Gamma_{R}=1, \theta=1$.

the spin torque components $T_{x, y, z}$ as a function of the static part of the adimensional barrier strength $z_{2}^{0}$ controlled by G2, while the remaining parameters have been fixed as follows: $z_{1}^{\omega}=z_{2}^{\omega}=0.2, \Gamma_{L}=0.9, z_{1}^{0}=0.5$, $a=1, \Gamma_{R}=1, \theta=1$. As shown, for increasing values of the static barrier strength $z_{2}^{0}$ the torque pumped in the system becomes vanishes. Indeed, the reduction of the spin current from the external leads reduces the gradient of the current and thus the spin torque is strongly suppressed. In the case of pumping, the suppression of the torque by increasing the barriers height may be in general stronger compared to the dc case. In Fig.(9) we show the spin torque components $T_{x, y, z}$ as a function of the Zeeman interaction $\Gamma_{L}$ for the other parameters: $z_{1}^{\omega}=z_{2}^{\omega}=0.2, z_{2}^{0}=1, z_{1}^{0}=0.5, a=1, \Gamma_{R}=1, \theta=1$. A behavior of the torque similar to the one presented in Fig. (5) is found. The magnetic interaction of the electron spins with the magnetic moment of M1 causes a suppression of the torque above $\Gamma_{L} \approx 1.7$ thus acting like an energy barrier for the electrons.

\section{CONCLUSIONS}

Within the scattering matrix approach we studied the spin torque generated by dc or ac external perturbations acting on a multilayered system consisting of a sequence of magnetic/nonmagnetic regions. In particular, we studied the spin-torque of a magnetic-nonmagnetic-magnetic trylayer connected to metallic nonmagnetic leads. We have focused on the effect of spin-filtering as the mechanism for current induced torque, i.e. the difference in the transmission and reflection probabilities for electrons with spins parallel and antiparallel to the moments of the magnetic layers. As a source of spin-dependent scattering we included also the spin-flip mechanism (i.e. the off diagonal elements of the scattering matrix). The spin torque generated by the application of a dc bias to the 


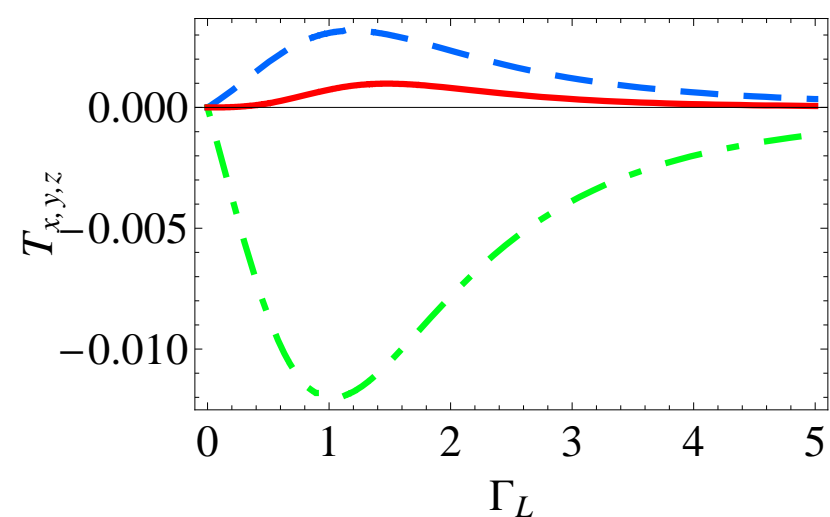

FIG. 9: Torque $T_{x}$ (dashed line), $T_{y}$ (dashed-dotted line), $T_{z}$ (full line) in unit of $\hbar \nu / 2$ computed as a function of $\Gamma_{L}$ controlled by the magnetic momentum in the region M1. The remaining parameters have been fixed as follows: $z_{1}^{\omega}=z_{2}^{\omega}=$ $0.2, z_{2}^{0}=1, z_{1}^{0}=0.5, a=1, \Gamma_{R}=1, \theta=1$.

external leads was analyzed as a function of the relative orientation $\theta$ between the magnetic moments of the magnetic regions, the length $2 a$ of the nonmagnetic spacer, the barriers transparencies and the Zeeman couplings. From the analysis we observed that the spin-torque vanishes when the magnetic moments of M1 and M2 are parallel or antiparallel, while an oscillating behavior of the spin-torque was observed as a function of $a$, indicating a quantum size effect. When the transparencies of the tunnel barriers at the interfaces between the mag- netic/nonmagnetic layers are lowered, a strong suppression of the spin torque is observed due to the reduction of the spin fluxes through the barriers. Similar effects were found as a function of the strength of the magnetic interaction $\Gamma_{L}$.

As an alternative to considering external dc bias, we proposed a current induced spin torque based on quantum pumping, generalizing the original idea of Thouless for the charges ${ }^{8}$. We formulated a scattering matrix formalism of the spin torque pumping. By modulating in time the strength of the two out-of-phase tunnel barriers at the interface between the magnetic/nonmagnetic layers, spin polarized currents were produced in the leads and a gradient of spin current responsible for the spin torque was generated. By studying the spin torque components in this case we observed different signatures of the magnetic reversal mechanism due to the pumping procedure. For instance, within the weak pumping limit additional harmonics were observed in the oscillations of $T_{\mu}$ vs the length of the nonmagnetic region $2 a$ and a stronger suppression of the spin torque at increasing the barrier heights. Thus the pumping mechanism could offer an additional tool to study experimentally the magnetic response of metallic heterostructures.

Experimentally, current induced spin torque has been realized in $\mathrm{Co} / \mathrm{Cu} / \mathrm{Co}$ sandwich structures ${ }^{15,16}$ and in $\mathrm{Py} / \mathrm{Cu} / \mathrm{Py}$ heterostructures 17 . Our proposal can also be realized by using two ferromagnetic EuS barriers coupled to $\mathrm{Al}$ leads and separated by an $\mathrm{Al}_{2} \mathrm{O}_{3}$ spacer of few tens of nanometers by modifying, for instance, the experimental setup studied in Ref.[18].
${ }^{1}$ For a review: Collection of articles in IBM J. Res. Dev. 42 (1998).

2 L. Berger, Phys. Rev. B 54, 9353 (1996).

3 J. Slonczewski, J. Magn. Magn. Mater. 159, L1 (1996).

${ }^{4}$ P. M. Krstajic̀, M. Keller and F. M. Peeters, Phys. Rev. B 77, 174428 (2008).

5 A. Brataas, Y.V. Nazarov, and G.E.W. Bauer, Phys. Rev. Lett. 84, 2481 (2000)

${ }^{6}$ X. Waintal, E. B. Myers, P. W. Brouwer, and D. C. Ralph, Phys. Rev. B 62, 12317 (2000).

7 K. Carva and I. Turek, Phys. Rev. B 76, 104409 (2007).

8 D. J. Thouless, Phys. Rev. B 27, 6083 (1983).

${ }^{9}$ P. Sharma and P. W. Brouwer, Phys. Rev. Lett. 91, 166801 (2003); see also arxiv: cond-mat/0306001v2.

10 M. Büttiker, Phys. Rev. B 46, 12485 (1992).

${ }^{11}$ K. Carva, I. Turek, arXiv:0903.4387v2 [cond-mat.mtrl-sci] (2009).
12 Yuhang Chen, Xuean Zhao, and You-Quan Li, Semicond. Sci. Technol. 19, 930 (2004).

13 A. Kalitsov, M. Chshiev, I. Theodonis, N. Kioussis, and W. H. Butler, Phys. Rev. B 79, 174416 (2009).

14 V. K. Dugaev, V. R. Vieira, P. D. Sacramento, J. Barnaś, M. A. N. Araújo, and J. Berakdar, Int. J. Mod. Phys. B 21, 1659 (2007).

15 J. A. Katine, F. J. Albert, R. A. Buhrman, E. B. Myers, and D. C. Ralph, Phys. Rev. Lett. 84, 3149 (2000).

16 E. B. Myers, D. C. Ralph, J. A. Katine, R. N. Louie, and R. A. Buhrman, Science 285, 867 (1999).

17 M. AlHajDarwish, H. Kurt, S. Urazhdin, A. Fert, R. Loloee, W. P. Pratt, Jr., and J. Bass, Phys. Rev. Lett. 93, 157203 (2004).

18 Guo-Xing Miao et al., Phys. Rev. Lett. 102, 076601 (2009). 